\title{
About Solution of the Nonlinear Generalized Abel Integral Equation
}

\author{
Burkhan Kalimbetov \\ Department of Mathematics Akhmet Yasawi university \\ Turkestan, Kazakhstan, \\ E-mail: burkhan.kalimbetov [AT] ayu.edu.kz
}

\begin{abstract}
As is known, many problems of electronics, nuclear physics, optics and astrophysics, etc. are described by the Abel integral equation of the first kind. In this paper we consider the nonlinear generalized Abel equation and show that its solution can be represented as an integral of a power function. It is shown that the constructed analytical solution and the symbolic solution obtained by means of the computer mathematics system Maple coincides, and their planar and spatial graphs are presented.
\end{abstract}

Keywords---- integral equation, Abel integral equation, tautochron problem, improper integral, Euler integral, computer mathematics system Maple.

\section{INTRODUCTION}

The Abel integral equations of the first kind are used in plasma diagnostics, physical electronics, nuclear physics, optics and astrophysics $[3,4,6]$. In its turn, real-world problems in scientific fields such as solid state physics, plasma physics, fluid mechanics, chemical kinetics, and mathematical biology are generally non-linear, and are described by nonlinear integral equations.

In this paper, we consider a nonlinear Volterra integral equation of the first kind with a weak kernel feature of the integral term

$$
y(t) \int_{0}^{t} \frac{y(s)}{(t-s)^{\alpha}} d s=a t^{\beta},
$$

where $y(t)$ - unknown function to be defined, $a, \alpha, \beta$-constants. We require performance of the following conditions for constants: $0<\alpha<1, \beta \geq 1-\alpha$ it is required to find non-trivial solution $y(t) \neq 0$ of the equation (1).

As is known, for the first time in the history of mathematics, an equation in which an unknown function $y(t)$ stood under the integral sign, except for the trivial problem

$$
\int_{0}^{t} y(x) d x=f(t)
$$

where $y(t)$ is given with an obvious solution

$$
y(t)=f^{\prime}(t)
$$

was obtained in 1826 by Abel when considering the tautochron problem [5]:

\section{THE MAIN RESULT}

Equation (1) is called the generalized Abel integral equation. Let us prove that it has a unique solution, which can be expressed in terms of the integral of the function $f(t)=a t^{\beta}$.

Suppose that it has a solution in the form $y(t)=k t^{\gamma}$, where $k, \gamma-$ constants to be defined. We put the function $y(t)=k t^{\gamma}$ into the equation (1),

$$
k t^{\gamma} \int_{0}^{t} \frac{\kappa s^{\gamma}}{(t-s)^{\alpha}} d s=a t^{\beta}
$$

Denote $s=t \tau$, then

$$
k^{2} t^{2 \gamma-\alpha+1} \int_{0}^{1} \frac{\tau^{\gamma}}{(1-\tau)^{\alpha}} d \tau=a t^{\beta},
$$


or

$$
k^{2} t^{2 \gamma-\alpha+1} \int_{0}^{1} \tau^{\gamma}(1-\tau)^{1-\alpha} d \tau=a t^{\beta} .
$$

Recall that the integral term of this equality is an improper integral of a special form. According to the theory of improper integrals, we obtain (see, e.g. [2], pp. 753-754):

$$
k^{2} t^{2 \gamma-\alpha+1} B(1-\alpha, 1+\gamma)=a t^{\beta},
$$

where

is Euler integral of the second kind.

$$
B\left(x_{1}, x_{2}\right)=\int_{0}^{1} \frac{d s}{(1-s)^{1-x_{1}} s^{1-x_{2}}}
$$

Equating here powers of the unknown variable $t$, we find the desired constants:

$$
\gamma=\frac{\beta+\alpha-1}{2}, \kappa= \pm a^{\frac{1}{2}} B^{-\frac{1}{2}}\left(1-\alpha, \frac{\beta+\alpha+1}{2}\right) \text {. }
$$

Thus, we have found a solution of the generalized Abel integral equation in the following form:

$$
y(t)= \pm a^{\frac{1}{2}} B^{-\frac{1}{2}}\left(1-\alpha, \frac{\beta+\alpha+1}{2}\right) t^{\frac{\beta+\alpha-1}{2}} .
$$

We formulate obtained result in the form of the following statement.

Theorem. If $f(t)=a t^{\beta}$ and $K(t, s)=(t-s)^{-\alpha}$ have continuous derivatives $f^{\prime}(t) \quad$ and $\frac{\partial K(t, s)}{\partial t}, f(0)=0, K(t, t) \neq 0$ on the segment $[0, T]$, then on $[0, T]$ the equation (1) has a unique continuous solution $y(t)$, representable as (3).

We check the program for finding a solution of the integral equation (1) in the case when the right-hand side is $f(t)=a t^{\beta}$, by using the system of computer mathematics Maple [1].

$>$ restart;

$>$ eq $1:=y(t) * \operatorname{int}\left((\mathrm{t}-\mathrm{s})^{\wedge}(-\right.$ alpha $\left.) * y(s), \mathrm{s}=0 . . \mathrm{t}\right)=\mathrm{a}^{*} \mathrm{t}^{\wedge}$ beta

$$
e q 1:=\mathrm{y}(t) \int_{0}^{t}(t-s)^{(-\alpha)} \mathrm{y}(s) d s=a t^{\beta}
$$

> eq2:=subs $\left(\mathrm{y}(\mathrm{t})=\mathrm{k}^{*} \mathrm{t}^{\wedge} \mathrm{c}, \mathrm{y}(\mathrm{s})=\mathrm{k}^{*} \mathrm{~s}^{\wedge} \mathrm{c}, \mathrm{eq} 1\right)$

$$
\begin{aligned}
& e q 2:=\frac{\sqrt{\Gamma(-\alpha+1) \Gamma\left(\frac{1}{2}+\frac{\alpha}{2}+\frac{\beta}{2}\right) a \Gamma\left(-\frac{\alpha}{2}+\frac{3}{2}+\frac{\beta}{2}\right)}}{\Gamma(-\alpha+1) \Gamma\left(\frac{1}{2}+\frac{\alpha}{2}+\frac{\beta}{2}\right)}, \\
& -\frac{\left.\sqrt{\Gamma(-\alpha+1) \Gamma\left(\frac{1}{2}+\frac{\alpha}{2}+\frac{\beta}{2}\right) a \Gamma\left(-\frac{\alpha}{2}+\frac{3}{2}+\frac{\beta}{2}\right)}\right)}{\Gamma(-\alpha+1) \Gamma\left(\frac{1}{2}+\frac{\alpha}{2}+\frac{\beta}{2}\right)} t^{\left(-1 / 2+\frac{\alpha}{2}+\frac{\beta}{2}\right)} \int_{0}^{t}(t-s)^{(-\alpha)} \\
& \frac{\sqrt{\Gamma(-\alpha+1) \Gamma\left(\frac{1}{2}+\frac{\alpha}{2}+\frac{\beta}{2}\right) a \Gamma\left(-\frac{\alpha}{2}+\frac{3}{2}+\frac{\beta}{2}\right)}}{\Gamma(-\alpha+1) \Gamma\left(\frac{1}{2}+\frac{\alpha}{2}+\frac{\beta}{2}\right)},
\end{aligned}
$$

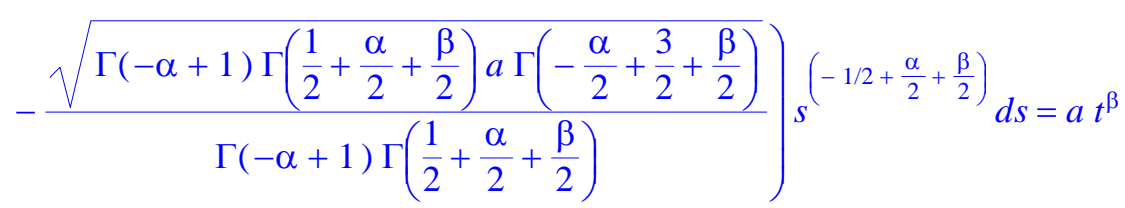


$>$ eq $3:=\mathrm{k}^{*} \mathrm{t}^{\wedge} \mathrm{c} * \operatorname{int}\left((\mathrm{t}-\mathrm{s})^{\wedge}(-\right.$ alpha $\left.) * \mathrm{k}^{*} \mathrm{~s}^{\wedge} \mathrm{c}, \mathrm{s}=0 . \mathrm{t}\right)=\mathrm{a} * \mathrm{t}^{\wedge}$ beta;

$$
e q 3:=k t^{c} \int_{0}^{t}(t-s)^{(-\alpha)} k s^{c} d s=a t^{\beta}
$$

$>\mathrm{c}:=\operatorname{solve}\left(\mathrm{t}^{\wedge} \mathrm{c}^{*} \mathrm{t}^{\wedge}(-\right.$ alpha $+1+\mathrm{c})=\mathrm{t}^{\wedge}$ beta, $\left.\mathrm{c}\right)$

$$
c:=-\frac{1}{2}+\frac{\alpha}{2}+\frac{\beta}{2}
$$

$>$ eq4: $=\operatorname{subs}(\mathrm{c}=-1 / 2+(1 / 2) *$ alpha $+(1 / 2) *$ beta, $\mathrm{t}=1$, eq 3$)$ :

$>\mathrm{k}:=\operatorname{solve}(\mathrm{eq} 4, \mathrm{k})$

$>\mathrm{y}(\mathrm{t}):=\mathrm{k}^{*} \mathrm{t}^{\wedge} \mathrm{c}$

$$
\begin{gathered}
y(t):=\left(\frac{\sqrt{\Gamma(-\alpha+1) \Gamma\left(\frac{1}{2}+\frac{\alpha}{2}+\frac{\beta}{2}\right) a \Gamma\left(-\frac{\alpha}{2}+\frac{3}{2}+\frac{\beta}{2}\right)}}{\Gamma(-\alpha+1) \Gamma\left(\frac{1}{2}+\frac{\alpha}{2}+\frac{\beta}{2}\right)},\right. \\
-\frac{\sqrt{\Gamma(-\alpha+1) \Gamma\left(\frac{1}{2}+\frac{\alpha}{2}+\frac{\beta}{2}\right) a \Gamma\left(-\frac{\alpha}{2}+\frac{3}{2}+\frac{\beta}{2}\right)}}{\Gamma(-\alpha+1) \Gamma\left(\frac{1}{2}+\frac{\alpha}{2}+\frac{\beta}{2}\right)} t\left(-1 / 2+\frac{\alpha}{2}+\frac{\beta}{2}\right)
\end{gathered}
$$

Graphs of the function (4) are given in Figure 1 - Figure 4.

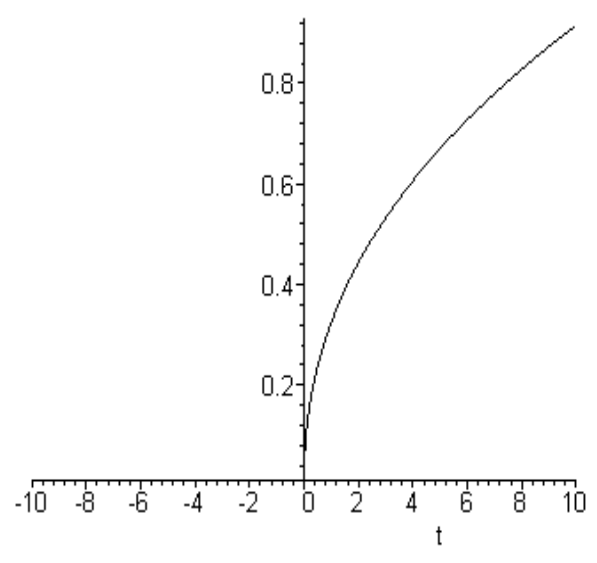

Figure 1: Function graph (two-dimensional case, when, $a=1 ; \alpha:=\frac{9}{10} ; \beta:=1 ; t=-10 . .10$ )

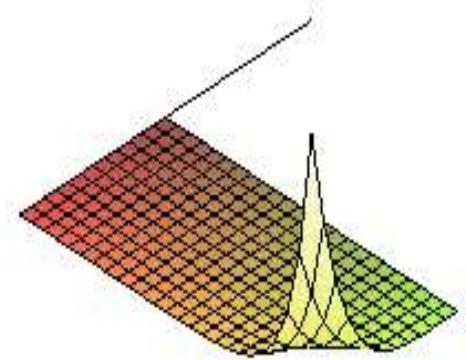

Figure 3: Function graph three-dimensional when $a=1 ; \alpha:=\frac{9}{10} ; \beta=\frac{1}{10} . .10$;

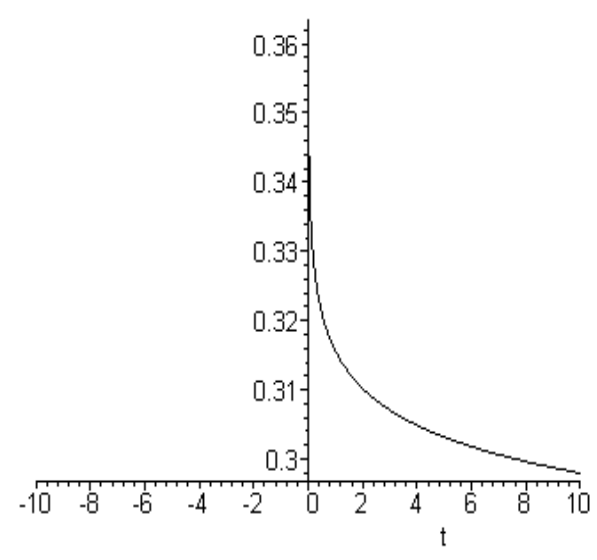

Figure 2: Function graph (two-dimensional case, when, $a=1 ; \alpha:=\frac{9}{10} ; \beta:=\frac{5}{100} ; t=-10 . .10$ )

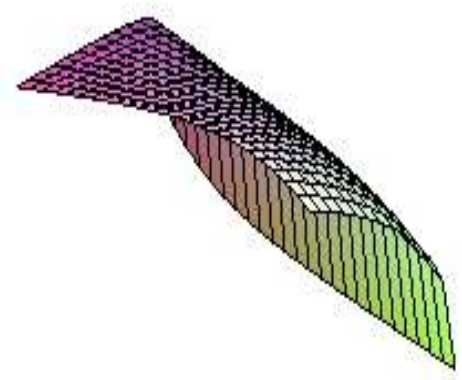

Figure4: Function graph three-dimensional case, case, when $a=1 ; \beta:=\frac{99}{10} ; \alpha=\frac{1}{10} . . \frac{8}{10}$;

Now we consider more general case, i.e. under the assumption that the solution of the integral equation (1) belongs to the class of continuous functions, $t^{\alpha} y(t) \in C[0, T], T>0$. 
First we replace the independent variable $t$ to $\tau$. Multiplying both sides of the equation by the factor $\frac{1}{(t-\tau)^{\alpha}}$, and taking integral from the obtained equality by the independent variable $\tau$ in the interval $[0, t]$, we have

$$
\int_{0}^{t} \frac{y(\tau)}{(t-\tau)^{\alpha}}\left(\int_{0}^{\tau} \frac{y(s) d s}{(\tau-s)^{\alpha}}\right) d \tau=\int_{0}^{t} \frac{a \tau^{\beta} d \tau}{(t-\tau)^{\alpha}}
$$

In relation (4) we introduce the formal transformation $\psi(\tau)=\int_{0}^{\tau} \frac{y(s, \varepsilon)}{(\tau-s)^{\alpha}} d s$. As a result, we get the equation:

$$
\int_{0}^{t} c \psi^{\prime}(\tau) \psi(\tau) d \tau=\int_{0}^{t} \frac{a \tau^{\beta} d \tau}{(t-\tau)^{\alpha}}
$$

Solving this equation, we get a solution concerning to the transformation $\psi(t)= \pm \sqrt{\frac{2}{c} \int_{0}^{t} \frac{a \tau^{\beta} d \tau}{(t-\tau)^{\alpha}}}$. Putting the found values into the equation (1), we obtain the generalized Abel integral equation:

$$
\int_{0}^{t} \frac{y(s)}{(t-s)^{\alpha}} d s= \pm \sqrt{\frac{2}{c} \int_{0}^{t} \frac{a s^{\beta} d s}{(t-s)^{\alpha}}}
$$

Solution of the equation (5) is a function

$$
y(t)=\frac{ \pm \sin \alpha \pi}{\pi} \frac{d}{d t} \int_{0}^{t} \frac{\sqrt{\frac{2}{c} \int_{0}^{\tau} \frac{a s^{\beta} d s}{(\tau-s)^{\alpha}}} d \tau}{(t-\tau)^{1-\alpha}}
$$

where $c=B\left(\frac{\beta-\alpha+1}{2}, \frac{\beta-\alpha+1}{2}\right) / B\left(\frac{\beta-\alpha+1}{2}, \frac{\beta+\alpha+1}{2}\right)$.

The obtained solutions (3) and (6) coincide.

Acknowledgments. This work is supported by the grant AP05133858 "Contrast structures in singularly perturbed equations and their application in the theory of phase transitions" by the Scientific Committee of the Ministry of Education and Science of the Republic of Kazakhstan.

\section{REFERENCES}

[1] Aladiev, V. (2006) Fundamentals of Programming in Maple, Tallinn.

[2] Fikhtengolts, G. (1969) Course of Differential and Integral Calculus, Vol. II, Moscow, Nauka.

[3] R. Gorenflo, R. and S. Vessella, S. (1991) Abel integral equations, Lecture Notes in Mathematics, 1461, Springer, Berlin.

[4] Kosarev, E. (1980) Applications of integral equations of the first kind in experiment physics, Comput. Phys. Commun. 20, 69-75.

[5] Muintz, G. (1934) Integral Equations. Part one. Linear Volterra equations, Moscow, GTTI.

]6] Plato, R. (2005) Fractional multistep methods for weakly singular Volterra integral equations of the first kind with perturbed data, Numer. Funct. Anal. Optim. 26, 249-269. 